CARDIOVASCULAR MEDICINE

\title{
Ventriculo-vascular interactions in patients with $\beta$ thalassaemia major
}

\author{
Y F Cheung, S Y Ha, G C F Chan
}

Heart 2005;91:769-773. doi: 10.1136/hrt.2003.032110

See end of article for authors' affiliations

Correspondence to:

Dr Y F Cheung, Division of

Paediatric Cardiology,

Department of Paediatrics

and Adolescent Medicine,

Grantham Hospital, The

University of Hong Kong,

125 Wong Chuk Hang

Road, Aberdeen, Hong

Kong, China; xf'cheung@

hkucc.hku.hk

Accepted 28 July 2004

\begin{abstract}
Objectives: To determine potential interactions between the heart and arterial system in patients with $\beta$ thalassaemia major.

Design and patients: Vascular compliance, systemic vascular resistance, and left ventricular (LV) contractility was determined in 34 asymptomatic thalassaemia patients at 2-4 hours after blood transfusion and also in 34 age and sex matched controls using a non-invasive device. The results were compared between groups and inter-relationships between LV contractility and indices of vascular load were explored.

Setting: Tertiary paediatric cardiac centre.

Results: When compared with controls, patients had greater systemic vascular resistance (1633 (259) v $1377(276)$ dynes $\left./ \mathrm{s} / \mathrm{cm}^{5}, \mathrm{p}<0.001\right)$ and effective arterial elastance $\left(E_{a}\right)(1.86(0.25) \quad v \quad 1.65$ (0.29) $\mathrm{mm} \mathrm{Hg} / \mathrm{ml}, \mathrm{p}=0.001$ ), an index of combined pulsatile and static vascular load. On the other hand, their systolic blood pressure (104 (9) v 112 (13) mm Hg, p=0.006), pulse pressure (45 (9) v 57 (10) $\mathrm{mm} \mathrm{Hg}, \mathrm{p}<0.001)$, adjusted systemic vascular compliance (1.21 (0.09) v 1.37 (0.14), $\mathrm{p}<0.001$ ), adjusted brachial artery distensibility (21 (0.29) $\vee 7.95(0.29) \% / \mathrm{mm} \mathrm{Hg}, \mathrm{p}<0.001)$ and LV+dP/dt (1059 (183) v 1239 (237) mm Hg/s, p = 0.001) were significantly lower. Significant determinants of LV contractility, as reflected by $\mathrm{LV}+\mathrm{dP} / \mathrm{dt}$, were age (standardised $\beta=-0.24, \mathrm{p}=0.003$ ), body mass index (standardised $\beta=-0.34, p=0.004$ ), systolic blood pressure (standardised $\beta=0.90$, $\mathrm{p}<0.001$ ), and effective $\mathrm{E}_{\mathrm{a}}$ (standardised $\beta=-0.50, \mathrm{p}<0.001$ ) (model $R^{2}=0.69$ ). No significant correlation existed between serum ferritin concentration and any of the cardiac or vascular indices.

Conclusion: An unfavourable ventriculo-vascular interaction, as characterised by impaired cardiac contractility and increased static and pulsatile vascular load, occurs in patients with $\beta$ thalassaemia major.
\end{abstract}

A bnormalities of left ventricular (LV) function are well documented in patients with $\beta$ thalassaemia major. ${ }^{12}$ In those with symptomatic heart failure, reduced LV shortening and ejection fractions are evident. Even in clinically asymptomatic patients, LV contractile dysfunction has been unmasked by dobutamine infusion. ${ }^{3}$ While myocardial iron deposition ${ }^{4}$ and myocarditis ${ }^{5}$ have a definite role in the pathogenesis of cardiac dysfunction, arterial dysfunction has also recently been shown by our group to be contributory. ${ }^{6}$

The relevance of arterial dysfunction becomes obvious when the heart and arterial system are considered from a mechanical perspective. Our finding of an increase in arterial stiffness in patients with $\beta$ thalassaemia major, ${ }^{6}$ which is probably related to structural alteration ${ }^{78}$ and endothelial dysfunction, suggests an increase in input impedance, and hence the arterial load, that is presented to the left ventricle. To date, however, no studies have been performed to determine potential interactions between the heart and arterial system in these patients. This in part may be related to the need for invasive assessment of ventriculo-arterial coupling by cardiac catheterisation. ${ }^{9}$ Recently, a non-invasive technology has been developed that allows estimation of the peak rate of positive $\mathrm{LV}$ pressure change $(\mathrm{LV}+\mathrm{dP} / \mathrm{dt})$ and derivation of vascular compliance and resistance (Dynapulse, Pulse Metric, Inc, San Diego, California). ${ }^{10}$

Using this non-invasive technique, we determined the vascular compliance, systemic vascular resistance, and $\mathrm{LV}+\mathrm{dP} / \mathrm{dt}$, an index of LV contractility, simultaneously in clinically asymptomatic patients with $\beta$ thalassaemia major and compared the results to those of age and sex matched control subjects. We further determined the interrelations between LV contractility and indices of vascular load in patients and controls.

\section{METHODS \\ Subjects}

Patients with $\beta$ thalassaemia major were recruited from the haematology clinic of Queen Mary Hospital. All of the recruited patients had normal LV shortening fraction and absence of valvar incompetence as documented by serial echocardiographic studies. Smokers and those with heart failure, systemic hypertension, and diabetes mellitus were excluded. None of the patients were on vasoactive medications at the time of the study. Healthy subjects matched for age and sex were recruited as controls. All subjects gave informed consent.

For thalassaemia patients, the study was performed at 2-4 hours after blood transfusion, so as to minimise the potentially confounding influence of anaemia and acute volume loading on the assessment results. Body weight and height were measured and the body surface area was calculated accordingly. In patients, the mean serum ferritin concentration was derived by averaging six to eight values obtained approximately every two months for 12 months.

\section{Non-invasive assessment of arterial pressure and vascular compliance}

Non-invasive arterial pressure signals were obtained with a Dynapulse 5200A instrument interfaced to a personal computer. The device records oscillometric pressure waveform signals from the brachial artery using an appropriately sized pressure cuff that was wrapped around the right upper arm. This technology uses a pulse dynamic pattern 
recognition methodology. ${ }^{10}$ The oscillometric signal is created by coupling pressure changes in the brachial artery to pressure changes in the cuff sphygmomanometer. As the cuff deflates from supra-systolic and sub-diastolic pressure, a silicon membrane pressure transducer produces an electric signal, which is then digitised using an 8-bit A/D converter for storage and analysis. The signals were stored in the hard disk of the personal computer and analysed offline using an automated system (Pulse Metric Inc) to derive the various cardiac and systemic vascular parameters. Three recordings were obtained from each of the subjects after at least 15 minutes of rest in the seated position, and the average was calculated for subsequent analyses.

\section{Cardiac parameters}

The cardiac output was calculated using proprietary algorithms and a model based on $\mathrm{LV}+\mathrm{dP} / \mathrm{dt}$, heart rate, and empirically derived scaling factors. The cardiac index was calculated as cardiac output/body surface area, and the stroke volume as cardiac output/heart rate.

The $\mathrm{LV}+\mathrm{dP} / \mathrm{dt}$ was derived from the arterial $+\mathrm{dP} / \mathrm{dt}^{11}$ assuming Gaussian curves for both aortic and LV wave contours. This method has been validated with simultaneous measurements using invasive micromanometer tipped catheters in both normal subjects and patients with myocardial infarction ( $r=0.75$ at baseline, $r=0.93$ with inotropes). ${ }^{12}$

\section{Vascular parameters}

The systolic and diastolic blood pressures were determined using standard oscillometric algorithms which have been shown to correlate with values obtained by auscultation using mercury cuff sphygmomanometer $(r=0.97$ for systolic pressure and $r=0.89$ for diastolic pressure). ${ }^{13}$

An index of systemic vascular compliance was calculated as: cardiac output/[heart rate-pulse pressure]. A good correlation $(r=0.83)$ has been demonstrated between this index and that derived by cardiac catheterisation. ${ }^{10}$ In addition, segmental brachial artery distensibility was derived from pressure waveform parameters, assuming a straight tube brachial artery and $\mathrm{T}$ tube aortic system, as: brachial arterial distensibility $\approx 4 \pi /\left(\mathrm{dP} / \mathrm{dt}_{\mathrm{pp}}-\mathrm{t}_{\mathrm{pp}}\right)$, where $\mathrm{dP} / \mathrm{dt}_{\mathrm{pp}}$ is the amplitude from peak positive pressure derivative to the peak negative pressure derivative, and $t_{p p}$ is the time interval between positive and peak negative pressure derivatives. ${ }^{14}$ This approximation holds true as the brachial artery diameter is much less than the cuff width. The systemic vascular resistance was derived as mean blood pressure/cardiac output.

To take into account both the steady and pulsatile components of the arterial load, the effective arterial elastance $\left(E_{a}\right),{ }^{15}$ which is the ratio of LV end systolic pressure

Table 1 Comparison of demographic data and blood pressure between patients and controls

\begin{tabular}{llll}
\hline & $\begin{array}{l}\text { Patients } \\
(\mathbf{n}=34)\end{array}$ & $\begin{array}{l}\text { Controls } \\
(\mathbf{n}=34)\end{array}$ & $\mathbf{p}$ Value \\
\hline Age (years) & $19.4(6.6)$ & $19.7(6.7)$ & 0.86 \\
Weight $(\mathrm{kg})$ & $41.5(11)$ & $53.3(14.8)$ & $<0.001^{*}$ \\
Height $(\mathrm{cm})$ & $150(13)$ & $160(13)$ & $0.002^{*}$ \\
Body mass index $\left(\mathrm{kg} / \mathrm{m}^{2}\right)$ & $18.0(2.5)$ & $20.4(3.8)$ & $0.004^{*}$ \\
Heart rate $($ beats $/ \mathrm{min})$ & $79(10)$ & $81(12)$ & 0.62 \\
Systolic BP $(\mathrm{mm} \mathrm{Hg})$ & $104(9)$ & $112(13)$ & $0.006^{*}$ \\
Diastolic BP $(\mathrm{mm} \mathrm{Hg})$ & $69(7)$ & $68(8)$ & 0.65 \\
Mean BP (mm Hg) & $80(8)$ & $82(9)$ & 0.34 \\
Pulse pressure $(\mathrm{mm} \mathrm{Hg})$ & $45(9)$ & $57(10)$ & $<0.001^{*}$ \\
\hline *Denotes significance. & & & \\
BP, blood pressure. & & & \\
\hline
\end{tabular}
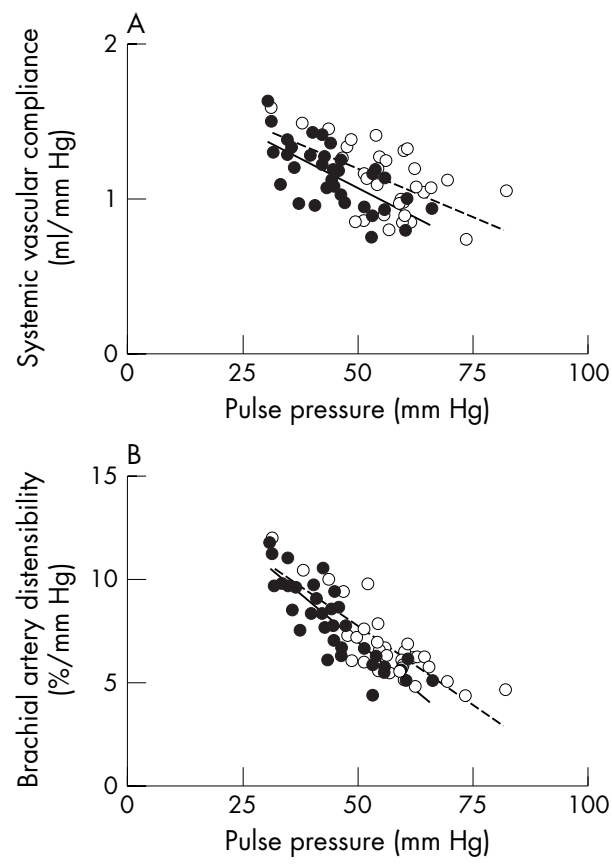

Figure 1 Scatter plots showing (A) significant negative correlation between systemic vascular compliance and pulse pressure $(r=-0.56$, $p<0.001)$, and (B) similar negative correlation between brachial artery distensibility and pulse pressure $(r=-0.85, p<0.001)$. The solid and dashed lines represent linear regression of vascular compliance and arterial distensibility on pulse pressure in patients (solid circles) and control subjects (open circles), respectively.

to stroke volume, was calculated. The LV end systolic pressure was approximated by the formula: $[2 \times$ systolic pressure + diastolic pressure ]/3. ${ }^{16}$

\section{Statistical analysis}

Data are presented as mean (SD) unless otherwise stated. Differences in demographic data, cardiac, and vascular parameters between patients and controls were compared using Student's $t$ test. Within the patient group, Pearson correlation analysis was used to assess for possible relations between serum ferritin concentration and various cardiovascular indices. Stepwise multiple linear regression was used to identify significant determinants of LV contractility. Significance was defined as $\mathrm{p}<0.05$. All statistical analyses were performed using SPSS Version 10.0 (SPSS, Inc, Chicago, Illinois).

\section{RESULTS}

\section{Patients}

Thirty four patients (18 males) were studied at an age of 19.4 (6.6) years. Their haemoglobin was $13.9(0.8) \mathrm{g} / \mathrm{dl}$ at the time of study, while their average ferritin concentration over the previous year was 5258 (2340) pmol/l. The demographic data and blood pressures of the patients and control subjects are summarised in table 1 . Thalassaemia patients had significantly smaller body size $(p<0.05)$, lower systolic blood pressure $(p=0.006)$, and smaller pulse pressure $(\mathrm{p}<0.001)$.

\section{Vascular parameters}

The cardiac and vascular parameters of patients and controls are summarised in table 2. When compared with control subjects, patients had significantly higher systemic vascular resistance $(p<0.001)$, and hence a greater static vascular load. The pulsatile vascular load was reflected by the indices 
of systemic vascular compliance and brachial artery distensibility. Given the significantly lower pulse pressure in patients and the known inverse relation between pulse pressure and vascular compliance and distensibility, adjustments of the latter indices were required for meaningful comparisons. ${ }^{14}$ After adjusting for the mean pulse pressure, ${ }^{14}$ patients were found to have significantly lower systemic vascular compliance $(p<0.001)$ and brachial artery distensibility $(\mathrm{p}=0.001)$.

The systemic vascular compliance and brachial artery distensibility were further analysed as a function of pulse pressure (fig 1). While the slopes of the two regression lines were similar (fig $1 \mathrm{~A}, \mathrm{p}=0.55$; fig $1 \mathrm{~B}, \mathrm{p}=0.23$ ), the elevations were significantly different (fig $1 \mathrm{~A}, \mathrm{p}=0.01$; fig $1 \mathrm{~B}, \mathrm{p}=0.008)$, suggesting that while systemic vascular compliance and brachial artery distensibility decreased with an increase in pulse pressure in both groups, patients had significantly lower vascular compliance and distensibility throughout the range of pulse pressure.

The effective $E_{a}$ was significantly greater in patients than in controls $(\mathrm{p}=0.001)$. This index correlated positively with total vascular resistance $(r=0.69, \mathrm{p}<0.001)$ and negatively with systemic vascular compliance $(r=-0.32$, $\mathrm{p}=0.008$ ).

There was no significant correlation between serum ferritin concentration and systemic vascular resistance $(p=0.42)$, adjusted systemic vascular compliance $(p=0.10)$, or adjusted brachial artery distensibility $(\mathrm{p}=0.81)$.

\section{Cardiac parameters}

The cardiac and stroke volume indices were similar between patients and controls (table 2). However, the LV+dP/dt was significantly lower in patients than in controls $(p=0.001)$. Multiple linear regression analysis of the entire cohort was used to identify significant determinants of $\mathrm{LV}+\mathrm{dP} / \mathrm{dt}$. The dependent variables included were: age, sex, body mass index, systolic and diastolic blood pressures, Ea, and patient status. Significant determinants were age (standardised $\beta=-0.24, p=0.003$ ), body mass index (standardised $\beta=-0.34, p=0.004$ ), systolic blood pressure (standardised $\beta=0.90, \quad p<0.001$ ), and Ea (standardised $\beta=-0.50, \mathrm{p}<0.001$ ) (model $R^{2}=0.69$ ). In patients, serum ferritin concentration did not correlate with either the cardiac index $(p=0.30)$ or $L V+d P / d t(p=0.36)$.

When relating $\mathrm{LV}+\mathrm{dP} / \mathrm{dt}$ to $\mathrm{E}_{\mathrm{a}}$ (fig 2 ), it became obvious that the cardiovascular system in patients with $\beta$ thalassaemia major was operating at a disadvantageous state, as characterised by diminished cardiac contractility and increased vascular load.

\section{DISCUSSION}

This study provides the first evidence of unfavourable interactions between the heart and arterial system in patients with $\beta$ thalassaemia major. Albeit asymptomatic and with

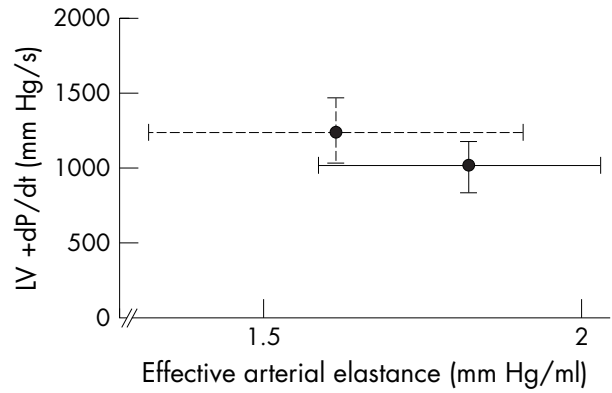

Figure 2 Unfavourable haemodynamic status of $\beta$ thalassaemia patients is characterised by reduced $L V+d P / d t$ and increased effective $E_{a}$ (solid lines), as compared to healthy subjects (dashed lines). Error bars represent mean (SD).

normal LV shortening fraction on serial echocardiographic evaluations, thalassaemia patients have significantly lower $\mathrm{LV}+\mathrm{dP} / \mathrm{dt}$ that suggests LV contractile dysfunction. Superimposed on this contractile abnormality is the increase in static and pulsatile vascular load. Notably, the myocardial contractility correlates inversely with the magnitude of the vascular load.

The index of myocardial contractility used in the present study was $\mathrm{LV}+\mathrm{dP} / \mathrm{dt}$, as estimated from the arterial $\mathrm{dP} / \mathrm{dt} .{ }^{11} \mathrm{As}$ aforementioned, the method has been validated with simultaneous measurements using invasive micromanometer tipped catheters. ${ }^{12}$ Our findings of reduced $\mathrm{LV}+\mathrm{dP} / \mathrm{dt}$ in thalassaemia patients corroborate those obtained in animal models of chronic iron overload, which showed a dose dependent decrease in $\mathrm{LV}+\mathrm{dP} / \mathrm{dt}$ in isolated perfused heart preparations. ${ }^{17}{ }^{18}$ While the exact mechanism whereby iron interferes with cardiac function remains unclear, disruption of intracellular calcium homeostasis ${ }^{19}$ and free radial mechanisms $^{18} 20$ have been implicated. The preservation of $\mathrm{LV}$ fractional shortening, despite the changes in $\mathrm{LV}+\mathrm{dP} / \mathrm{dt}$, has likewise been observed in the animal model. ${ }^{17}$ This discrepancy is probably related to the load sensitive nature of the index of LV shortening fraction and the masking of underlying contractile dysfunction as a result of the increase in LV mass present in both iron overloaded patients ${ }^{6}$ and animal models. ${ }^{17}$ Indeed, abnormal myocardial longitudinal function has been demonstrated by Doppler tissue imaging in thalassaemia patients whose conventional indices of global systolic function are within normal. ${ }^{21}$

While cardiac dysfunction has been well documented in patients with $\beta$ thalassaemia major, ${ }^{2}$ dysfunction of the arterial system, manifested as increased arterial stiffness and endothelial dysfunction, has only recently been unveiled by our group. ${ }^{6}$ The present findings in patients of a lower systemic vascular compliance and brachial arterial distensibility, using a different methodology, further confirm the

Table 2 Comparison of cardiac and vascular parameters between patients and controls

\begin{tabular}{llll}
\hline & Patients $(\mathbf{n}=34)$ & Controls $(\mathbf{n}=34)$ & $\mathrm{p}$ Value \\
\hline Cardiac index $\left(\mathrm{l} / \mathrm{min} / \mathrm{m}^{2}\right)$ & $3.13(0.61)$ & $3.24(0.64)$ & 0.48 \\
Stroke volume index $\left(\mathrm{ml} / \mathrm{min} / \mathrm{m}^{2}\right)$ & $38.4(3.2)$ & $39.3(3.8)$ & 0.28 \\
$\mathrm{LV}+\mathrm{dP} / \mathrm{dt}(\mathrm{mm} \mathrm{Hg} / \mathrm{s})$ & $1059(183)$ & $1239(237)$ & $0.001^{*}$ \\
$\begin{array}{l}\text { Systemic vascular resistance }\left(\text { dynes } / \mathrm{s} / \mathrm{cm}^{5}\right) \\
\text { Systemic vascular compliance, adjusted for mean pulse }\end{array}$ & $1.21(0.09)$ & $1377(276)$ & $<0.001^{*}$ \\
pressure $(\mathrm{ml} / \mathrm{mm} \mathrm{Hg})$ & $1.37(0.14)$ & $<0.001^{*}$ \\
Brachial artery distensbility, adjusted for mean pulse & $7.21(1.29)$ & $7.95(0.29)$ & $<0.001^{*}$ \\
$\begin{array}{l}\text { pressure }(\% / \mathrm{mm} \mathrm{Hg}) \\
\text { Effective } \mathrm{E}_{\mathrm{a}}(\mathrm{mm} \mathrm{Hg} / \mathrm{ml})\end{array}$ & $1.86(0.25)$ & $1.65(0.29)$ & $0.001^{*}$ \\
\hline *Denotes significance. & & &
\end{tabular}


results of our previous study. ${ }^{6}$ The resultant increase in pulsatile arterial load may be attributable to structural alterations in the arteries of thalassaemia patients ${ }^{7}$ and increased vasomotor tone secondary to endothelial dysfunction. ${ }^{6}$

Additionally, systemic vascular resistance, the static component of the vascular load, is also found to be increased in our patients when assessed within the first 24 hours of blood transfusion. This is in contrast to the previous findings of a low systemic vascular resistance in patients studied just before blood transfusion. ${ }^{22}{ }^{23}$ Nonetheless, the concomitant findings of increases in LV end systolic meridional and circumferential wall stress, indices of the ventricular afterload, in the previous studies are intriguing. ${ }^{22}{ }^{23}$ Explanations for the discrepant findings of a low systemic vascular resistance and a high LV afterload have not, however, been provided. Undoubtedly, patients with chronic anaemia have a high cardiac output state caused by a low systemic vascular resistance; this has been hypothesised to result from vasodilation secondary to reduced inhibition of endothelium derived relaxing factor by low circulating haemoglobin. ${ }^{24}$ Nonetheless, rapid normalisation of cardiac index and systemic vascular resistance has been documented after correction of severe chronic anaemia. ${ }^{24}$ Expectedly, the cardiac index became normalised in our patients after blood transfusion. It is intriguing, however, to find an elevated systemic vascular resistance in the early post-transfusion period, which, in conjunction with the increase in pulsatile vascular load, may account for the higher LV afterload observed in patients with $\beta$ thalassaemia major. While the exact cause remains unknown, arterial endothelial dysfunction may perhaps also explain the abnormally high systemic vascular resistance that may otherwise have been masked during the state of anaemia.

Hence, data from this and other studies ${ }^{22} 23$ suggest that the LV afterload is chronically increased in patients with $\beta$ thalassaemia major, the magnitude of which may perhaps be even higher in the early post-transfusion period. The implications, in the light of the ventricular-arterial coupling framework, are obvious. ${ }^{\text {" We }}$ have demonstrated that effective $\mathrm{E}_{\mathrm{a}}$, an index of combined pulsatile and static load, is a significant negative determinant of cardiac contractility. Thus, patients with $\beta$ thalassaemia major, albeit asymptomatic, have disadvantageous cardiovascular haemodynamics as characterised by impaired cardiac contractility and increased vascular load. While the aetiology of myocardial dysfunction cardiac function is multifactorial, ${ }^{4}$ the elevated vascular load may jeopardise the cardiac function further through suboptimal ventriculo-vascular coupling. Indeed, afterload reduction with oral enalapril has been shown to improve LV systolic and diastolic function in asymptomatic or minimally symptomatic thalassaemia patients with LV dysfunction. ${ }^{25}$

The finding of a low systolic blood pressure in thalassaemia patients agrees with that reported previously. ${ }^{26}$ Proposed explanations include a lower body mass index and reduced sympathetic activity secondary to stimulation of thoracic mechanoreceptors after blood transfusion..$^{25}$ The latter may perhaps also be envisaged as a compensatory mechanism for the increase in vascular load.

Determination of ventriculo-arterial coupling within the framework as advocated by Sunagawa and colleagues is not feasible because of the non-invasive nature of the cardiovascular assessment. ${ }^{15} 27$ Ideally, determination of the LV end systolic pressure volume relation, Ea, and aortic input impedance by invasive catheterisation studies will enable a more accurate assessment of coupling efficiency and LV afterload, respectively. Nonetheless, the results of the present study do suggest that the interaction between the heart and the arterial system is unfavourable in patients with $\beta$ thalassaemia major. Prophylactic vasodilator treatment in clinically asymptomatic patients may perhaps delay the deterioration of cardiac function in addition to iron chelation therapy.

\section{ACKNOWLEDGEMENTS}

The work described in this paper was fully supported by grants from the Research Grants Council of the Hong Kong Special Administrative Region, China (Project no. HKU 7233/01M).

\section{Authors' affiliations}

Y F Cheung, Division of Paediatric Cardiology, Grantham Hospital, University of Hong Kong, Hong Kong, PRC

S Y Ha, G C F Chan, Division of Paediatric Haematology and Oncology, Queen Mary Hospital, University of Hong Kong, Hong Kong, PRC

\section{REFERENCES}

1 Lewis BS, Rachmilewitz EA, Amitai N, et al. Left ventricular function in $\beta$ thalassemia and the effect of multiple transfusion. Am Heart $J$ 1978;96:636-45.

2 Kremastinos DT, Tsiapras DP, Tsetsos GA, et al. Left ventricular diastolic Doppler characteristics in beta-thalassemia major. Circulation 1993;88: 1127-35.

3 Hui L, Leung MP, Ha SY, et al. Early detection of left ventricular dysfunction in patients with beta-thalassemia by dobutamine stress echocardiography. Heart 2003:89:669-70.

4 Jessup M, Manno CS. Diagnosis and management iron-induced heart disease in Cooley' anemia. Ann N Y Acad Sci 1998;850:242-50.

5 Kremastinos DT, Tiniakos G, Theodorakis GN, et al. Myocarditis in betathalassemia major: a cause of heart failure. Circulation 1995;91:66-71.

6 Cheung YF, Chan GCF, Ha SY. Arterial stiffness and endothelial function in patients with beta-thalassaemia major. Circulation 2002;106:2561-6.

7 Tsomi K, Karagiorga-Lagana M, Fragodimitri C, et al. Arterial elastorrhexis: manifestation of a generalized elastic tissue disorder in beta-thalassemia major. Eur J Haematol 1999;63:287-94.

8 Cardoso LE, Mourao PA. Compositional and structural alterations of arterial glycosaminoglycans associated with the complications brought about by thalassemia major. A case report. Angiology 1996;47:175-83.

9 Kass DA, Kelly RP. Ventriculo-arterial coupling: concepts, assumptions, and applications. Ann Biomed Eng 1992;20:41-62.

10 Brinton TJ, Cotter B, Kailasam MT, et al. Development and validation of a noninvasive method to determine arterial pressure and vascular compliance. Am J Cardiol 1997;80:323-30.

11 Germano G, Angotti S, Muscolo $M$, et al. The (dP/dt) max derived from arterial pulse waveforms during $24 \mathrm{hr}$ blood pressure oscillometric recording. Blood Press Monitor 1998;3:213-6.

12 Brinton TJ, Hu WC, Chio SS, et al. The development and validation of a new non-invasive method to evaluate ventricle function during routine blood pressure monitoring. Am J Hypertens 1997; 10:60A.

13 Brinton TJ, Walls ED, Chio SS. Validation of pulse dynamic blood pressure measurement by auscultation. Blood Press Monitor 1998;3:121-4.

14 Urbina EM, Brinton TJ, Elkasabany A, et al. Brachial artery distensibility and relation to cardiovascular risk factors in healthy young adults (the Bogalusa heart study). Am J Cardiol 2002;89:946-51.

15 Sunagawa K, Maughan WL, Burkhoff D, et al. Left ventricular interaction with arterial load studied in isolated canine ventricle. Am J Physiol 1983:56:586-95.

16 Kelly RP, Ting CT, Yang TM, et al. Effective arterial elastance as index of arterial vascular load in humans. Circulation 1992;86:513-21.

17 Yang T, Dong WQ, Kuryshev YA, et al. Bimodal cardiac dysfunction in an animal model of iron overload. J Lab Clin Med 2002;140:263-71.

18 Bartfay WJ, Dawood F, Wen WH, et al. Cardiac function and cytotoxic aldehyde production in a murine model of chronic iron-overload. Cardiovasc Res 1999;43:892-900.

$19 \operatorname{Kim}$ E, Giri SN, Pessah IN. Iron (II) is a modulator of ryanodine-sensitive calicum channels of cardiac muscle sarcoplasmic reticulum. Toxicol Applied Pharmacol 1995; 130:57-66.

20 Livrea MA, Tesoriere L, Pintaudi AM, et al. Oxidative stress and antioxidant status in bete-thalassemia major: iron overload and depletion of lipid-soluble antioxidants. Blood 1996;88:3608-14.

21 Vogel M, Anderson $\amalg$, Holden S, et al. Tissue Doppler echocardiography in patients with thalassemia detects early myocardial dysfunction related to myocardial iron overload. Eur Heart J 2003;24:113-9.

22 Vaccari M, Crepaz R, Fortini $M$, et al. Left ventricular remodeling, systolic function, and diastolic function in young adults with $\beta$-thalassemia intermedia. Chest 2002;121:506-12.

23 Bosi G, Crepaz R, Gamberini MR, et al. Left ventricular remodelling, and systolic and diastolic function in young adults with $\beta$-thalassemia major: a Doppler echocardiographic assessment and correlation with haematological data. Heart 2003:89:762-6.

24 Anand IS, Chandrashekhar Y, Wander GS, et al. Endothelium-derived relaxing factor is important in mediating the high output state in chronic severe anemia. J Am Coll Cardiol 1995;25:1402-7. 
25 Karvounis HI, Zaglavara TA, Parharidis GE, et al. An angiotensin-converting enzyme inhibitor improves left ventricular systolic and diastolic function in transfusion-dependent patients with $\beta$-thalassemia major. Am Heart $J$ $2001 ; 141: 281$
26 Veglio F, Melchio R, Rabbia F, et al. Blood pressure and heart rate in young thalassemia major patients. Am J Hypertens 1998;1 1:539-47.

27 Sunagawa K, Sagawa K, Maughan WL. Ventricular interaction with the loading system. Ann Biomed Eng 1984;12:163-89.

\section{IMAGES IN CARDIOLOGY}

\section{Leff ventricular pseudoaneurysm in a child}

A 14 year old girl presented with a history of fatigue, atypical chest pain, and breathlessness for one month. Her haemoglobin was $8.3 \mathrm{~g} / \mathrm{dl}$ and erythrocyte sedimentation rate (ESR) was $48 \mathrm{~mm}$ in the first hour. She had an anterolateral myocardial infarction on ECG. Her echocardiogram revealed a large pseudoaneurysm of the mid and apical lateral left ventricular free wall. On cardiac catheterisation, the presence of large anterolateral left ventricular pseudoaneurysm was confirmed (panel A). It also showed constriction of the aortic arch, with a gradient of $30 \mathrm{~mm} \mathrm{Hg}$ across it, ectatic changes in arch vessels, and diffuse irregularity of the descending thoracic and abdominal aorta (panel B). Coronary angiogram revealed distal left anterior descending artery occlusion. The patient was diagnosed as having active non-specific aortoarteritis with involvement of coronary arteries with myocardial

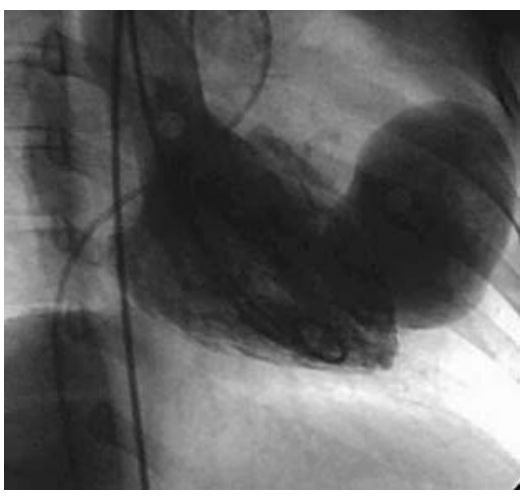

Left ventriculogram in right anterior oblique position showing presence of a large anterolateral pseudoaneurysm.

infarction. She underwent resection of the pseudoaneurysm and was started on steroids. The resected tissue revealed ischaemic changes. She was doing well at three month follow up.

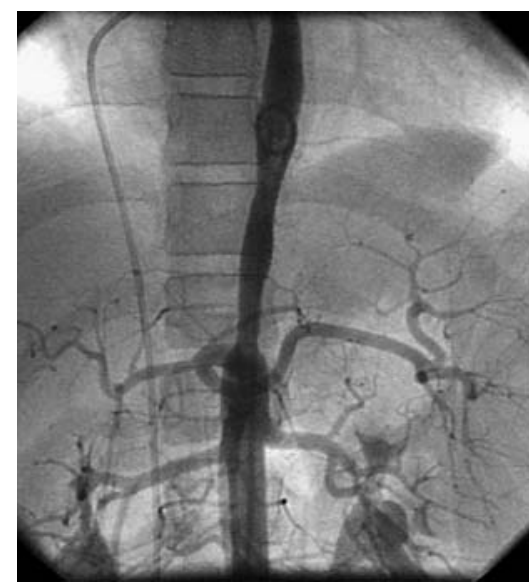

Aortogram showing diffuse irregularity of the descending thoracic aorta and upper abdominal aorta.

S S Kothari, A Roy, G Sharma kotharis@vsnl.net.in

A vascular ring without compression: double aortic arch presenting as a coincidental finding during cardiac catheterisation

W

e present a case of a 73 year old woman with double aortic arch and separate origin of the right common carotid and right subclavian arteries, found coincidentally during cardiac angiography. She has a history of coronary artery disease and atherosclerotic renal artery occlusion. Medical history includes indigestion (relieved by proton pump inhibition), deep vein thrombosis, pulmonary embolic disease, coronary bypass grafting, and a minor stroke. The image (three dimensional reconstructed magnetic resonance contrast angiography) demonstrates the double aortic arch. The right common carotid and right subclavian arteries arise separately from the posterior arch and the left common carotid and left subclavian from the anterior arch. The right and left vertebral arteries arise from the corresponding subclavian arteries. The right vertebral artery is stenotic and shows post-stenotic dilatation. Both aortic lumina are similar in calibre and intact. There was no dysphagia or stridor and there was no tracheal compression identified on native images. This observation suggests that the incidence and prevalence of this rare condition may be underestimated.

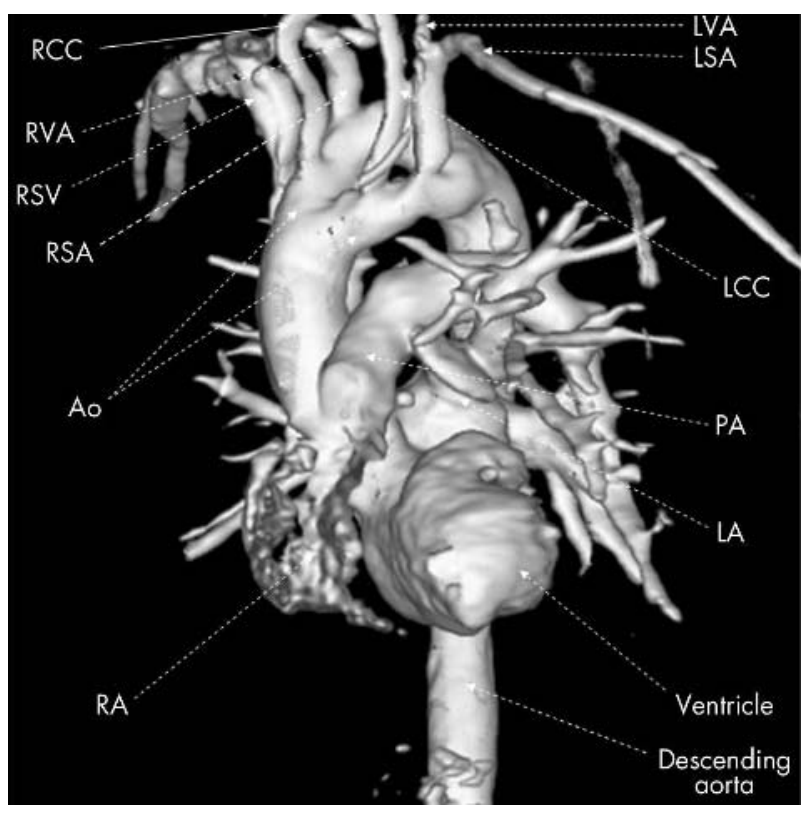

Ao, aorta; LCC, left common carotid; LSA, left subclavian artery; LVA, left vertebral artery; RCC, right common carotid; RSA, right subclavian artery; RVA, right vertebral artery. 